\title{
Preparation and Performance of Nickel Oxide Films by Ion Beam Sputtering Deposition and Oxidation Annealing
}

\author{
Jing Peng ${ }^{1,4}$, Zhimou $\mathrm{Xu}^{2,3, *}$, Shuangbao Wang ${ }^{2,3}$, Quanlin Jie ${ }^{1}$ and Cunhua $\mathrm{Chen}^{5}$ \\ ${ }^{1}$ Physical Science and Technology Institute, Wuhan University, Wuhan 430072, China \\ ${ }^{2}$ Wuhan National Laboratory for Optoelectronics, Wuhan 430074, China \\ ${ }^{3}$ College of Optoelectronic Science and Engineering, Huazhong University of Science and Technology, \\ Wuhan 430074, China \\ ${ }^{4}$ College of Sciences, Wuhan University of Science and Technology, Wuhan 430081, China \\ ${ }^{5}$ School of Chemistry, Central China Normal University, Wuhan 430079, China
}

(Received August 11, 2009; accepted February 5, 2010)

Key words: nickel oxide thin films, ion beam sputtering, oxidation annealing, microstructure

Polycrystalline $\mathrm{NiO}_{x}$ thin films were deposited on quartz substrates by ion beam sputter deposition and oxidation annealing at high temperatures. X-ray diffraction (XRD) and scanning electron microscopy (SEM) morphologies indicate that the as-deposited nickel oxide thin films are flat amorphous nickel or oxides. However, they developed into semiconducting $\mathrm{NiO}_{x}$ thin films during the oxidation annealing process. Four-point probe tests confirmed their sheet resistance and the resistance-temperature relationship. In addition, infrared (IR) measurements were also carried out in the visible spectrum range to study the optical properties of the as-deposited and annealed films. Apparently, the $\mathrm{NiO}_{x}$ films obtained by the new method exihibit properties that are comparable to those of the films formed by other methods. The thin films have potential application as gas sensors.

\section{Introduction}

Recently, there has been considerable public interest in our environment and its impact on the quality and quantity of life. The fast growth in automobile traffic and the high levels of emissions from industries in particular have increased research and development activities including those on gas sensors. Recently, semiconductor gas sensors have rapidly been developed at home and abroad for unique advantages, such as high sensitivity, high response speed, low cost and wide application. Semiconductor gas sensors use the chemical sensitivity of semiconductor surfaces for gas-sensing applications. Metal oxide semiconductors prepared in thin-film form ${ }^{(1-3)}$ have good sensitivity, optimum operating temperature and better selectivity in comparison with "Corresponding author: e-mail: xuzhimou2001@yahoo.com.cn 
conventional bulk gas sensors. However, there are few papers published on the preparation and characterization of $\mathrm{NiO}$ thin films in connection with their gas-sensing

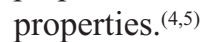

Nickel oxide $\left(\mathrm{NiO}_{x}\right)$ is an attractive material for use as an antiferromagnetic layer, ${ }^{(6)}$ and a p-type transparent semiconducting film ${ }^{(7)}$ and in electrochromic devices. ${ }^{(8)}$ Bulk $\mathrm{NiO}$ has a cubic (NaCl-type) structure with a lattice parameter of $0.4195 \mathrm{~nm}$. It is considered to be a model/typical semiconductor with hole-type conductivity. $\mathrm{NiO}_{x}$ thin films have been fabricated by various physical and chemical vapor deposition techniques, such as reactive sputtering and plasma-enhanced chemical vapor deposition, because the chemical stability of the layers as well as their optical and electrical properties are excellent.

It is well known that the structural properties and surface morphology of thin-films depend on the deposition techniques, deposition conditions and postdeposition processes. These properties of metal oxide films have become of great interest in the last few years. In particular, the field of gas sensors has benefited from the preparation of materials characterized by a high surface-to-volume ratio. ${ }^{(9)}$ The gas-sensing properties of metal oxides are more or less related to the material surface, its high porosity and benefits from a microstructure with a small particle size.

The effects of ion beam sputtering conditions (oxygen content in the gas mixture and pumping speed) and postdeposition annealing on the structural properties and surface morphology of $\mathrm{NiO}_{x}$ thin films are studied. The main objective of this work is to find the correlation between the process parameters and physical properties.

\section{Procedure}

$\mathrm{NiO}_{x}$ films were deposited by reactive ion beam sputtering from a $\mathrm{Ni}$ target $(80 \mathrm{~mm}$ in diameter, $5 \mathrm{~mm}$ in thickness, and normally $99.95 \%$ in purity) in a mixture of oxygen and argon on unheated quartz substrates. The distance between the target and the substrate was approximately $70 \mathrm{~mm}$. The details of the apparatus are given elsewhere. ${ }^{(10)}$ The chamber was evacuated to a pressure below $1 \times 10^{-3} \mathrm{~Pa}$ before deposition. A sputtering power of $40 \mathrm{~W}$ was used. Inert $\mathrm{Ar}$ and reactive oxygen were adjusted using mass flow controllers. The partial pressure of $\mathrm{O}_{2}$ was changed from $1 \times 10^{-3}$ to $1 \times 10^{-2} \mathrm{~Pa}$. The total gas pressure was kept at $2.5 \times 10^{-2} \mathrm{~Pa}$. Before deposition, the target was presputtered to remove contaminants. Then, $\mathrm{NiO}_{x}$ films were prepared by ion beam sputtering in the process. The ion beam sputtering conditions are listed in Table 1. After deposition, the films were annealed in a tube furnace for about $2 \mathrm{~h}$ in flowing $\mathrm{O}_{2}$ gas. The annealing temperatures were 400,500 , and $600^{\circ} \mathrm{C}$. The annealing apparatus is shown in Fig. 1.

The crystalline structure of $\mathrm{NiO}_{x}$ films was identified with Theta Diffractometer D5000 with copper K-alpha radiation. The surface morphology was observed by scanning electron microscopy (SEM).

Films for measuring the gas-sensing properties were cut into the size of $5 \times 5$ $\mathrm{mm}^{2}$, where the deposition of a $\mathrm{NiCr}$ alloy electrode and alloying were performed. The measurements were carried out in a semiclosed quartz tube oven that works in flowing gas mode. The gas chosen for measurement was $\mathrm{NH}_{3}(99.5 \%$ pure). The 
Table 1

Deposition conditions of the $\mathrm{NiO}_{x}$ films.

\begin{tabular}{ll}
\hline Parameter & Value \\
\hline Base pressure & $<1 \times 10^{-3} \mathrm{~Pa}$ \\
Oxygen pressure & $1-2 \times 10^{-3} \mathrm{~Pa}$ \\
Ion energy & $500 \mathrm{eV}$ \\
Beam current & $80 \mathrm{~mA}$ \\
Deposition period & $5 \mathrm{~min}$ \\
Substrate temperature & $200^{\circ} \mathrm{C}$ \\
\hline
\end{tabular}

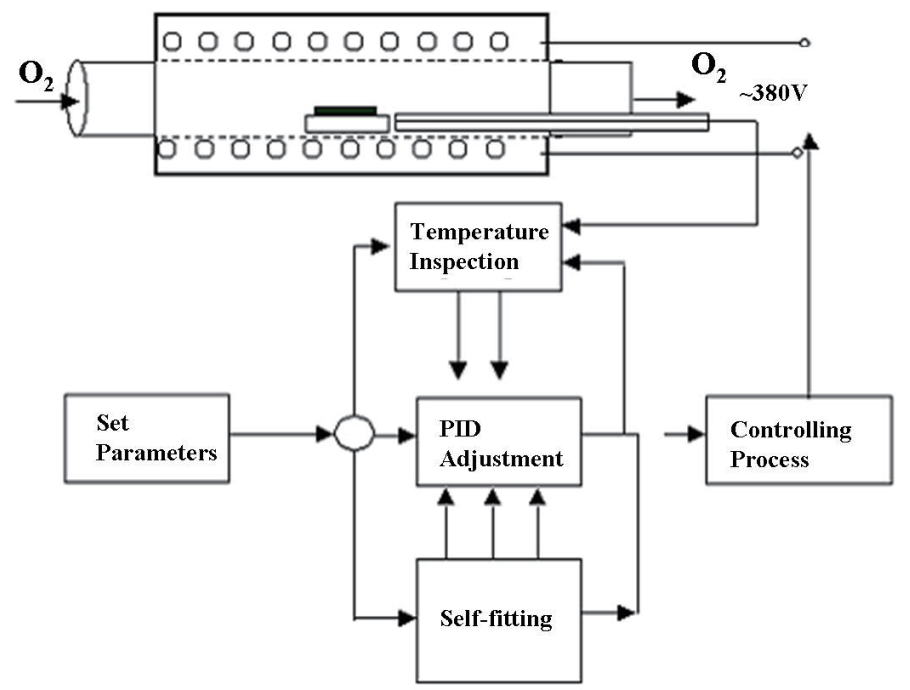

Fig. 1. Schematic diagram of the annealing chamber.

operating temperature was controlled in the range from 25 to $250^{\circ} \mathrm{C}$ with a Eurotherm thermoregulator. A DC voltage was applied across the sensing film and the current was measured using an HP 4140B electrometer (Hawplet Co. Ltd., USA).

\section{Results and Discussion}

\subsection{Structure}

Figure 2 shows the XRD pattern of the as-deposited and annealed samples by ion beam sputtering deposition. For the as-deposited samples, the data indicate that the thin films contain amorphous or very fine grains for there is only a wide amorphous peak covering the reflections of metallic nickel and/or any oxides. However, for the annealed samples, there are also some peaks corresponding to the reflections of $\mathrm{NiO}$ (111). The $\mathrm{NiO}$ reflections are more apparent in the XRD patterns of the samples annealed at high temperatures. Therefore, most nickel oxide grains have a lattice image with a preferential orientation of (111) crystal planes and only a few grains have a lattice image 


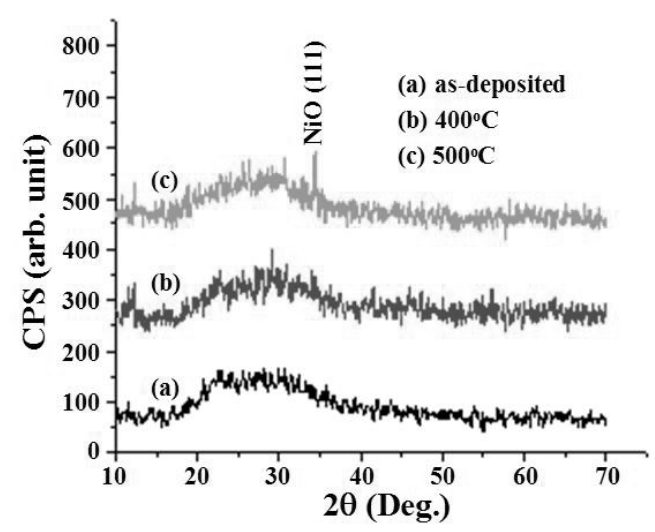

Fig. 2. XRD patterns of the as-deposited and annealed nickel oxide films.

showing (002) planes. From other works, ${ }^{(10)} \mathrm{NiO}_{x}$ is a stoichiometric phase that changes its composition at different oxidation levels. In fact, the reflections of $\mathrm{NiO}_{x}$ also shift slightly when annealing at different temperatures, possibly resulting from the change in composition with oxidation level. The as-deposited samples are expected to have low oxygen contents; thus, any nickel oxides formed are below the resolution of the XRD.

Figures 3(a) and 3(b) respectively show the surface and cross section morphologies of the annealed samples, obtained by SEM. The film thickness is about $165 \mathrm{~nm}$. The samples were deposited and annealed in an oxidation ambient for $2 \mathrm{~h}$. At this stage, the grains are large and compact and the surface roughness is large compared with those of the as-deposited samples.

The annealed samples are typically not homogeneous and their reproducibility is also low. In fact, the annealing is complex and there are many differences arising from changes in the as-deposited film component or annealing temperature distribution. For example, oxygen diffuses inwards along grain boundaries more easily than across grains. Therefore, the oxygen component is observed at different places. The final products are very different even under the same annealing conditions, and variable values of resistances are typically found after annealing. The values differ at different regions of the same sample.

\subsection{Properties}

The electrical resistivity and optical properties of nickel oxide thin films were examined. The annealing process was found to have a profound effect on the electrical resistivity. It reduces the resistivity with increasing annealing temperature, as shown in Fig. 4. The reduction in resistivity is due to the increased carrier concentration.

Figure 4 shows the resistance-temperature relationship curve of the nickel oxide thin films fabricated by ion beam sputtering and annealed at different temperatures for $2 \mathrm{~h}$. From these relationship curves, the tempature coefficient of resistance (TCR) values could be calculated from their slopes as $T C R=\mathrm{d} R / R \mathrm{~d} T$. The left figure (Fig. 4(a)) shows 


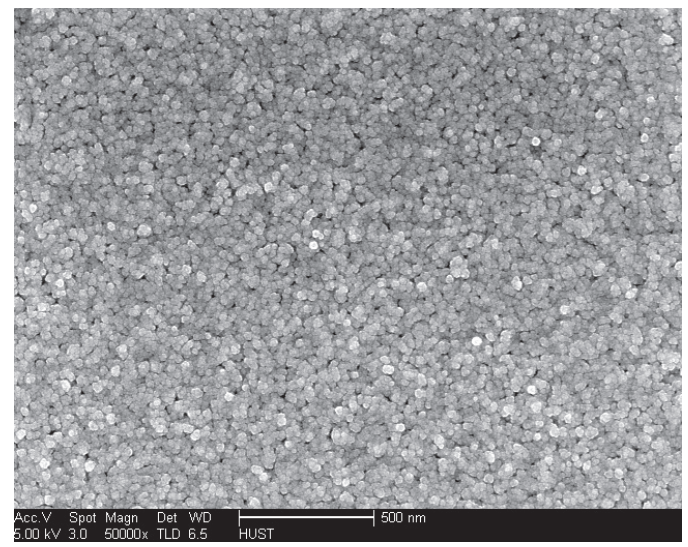

(a)
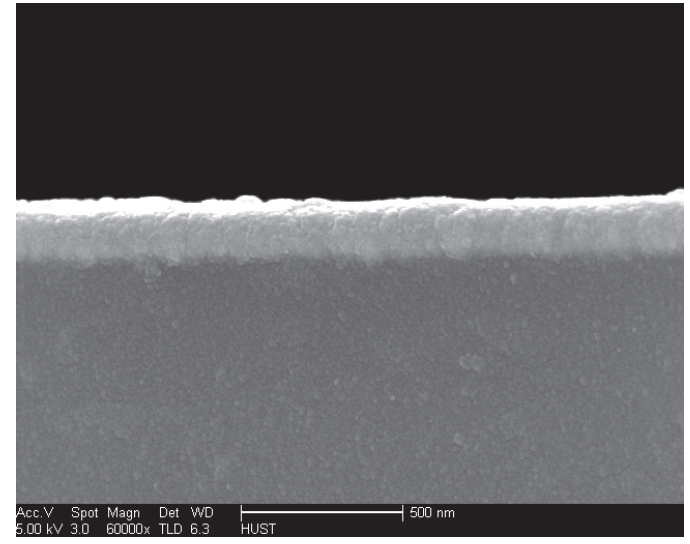

(b)

Fig. 3. SEM morphology of samples annealed for $2 \mathrm{~h}$ at $400^{\circ} \mathrm{C}$ : (a) surface and (b) cross section.

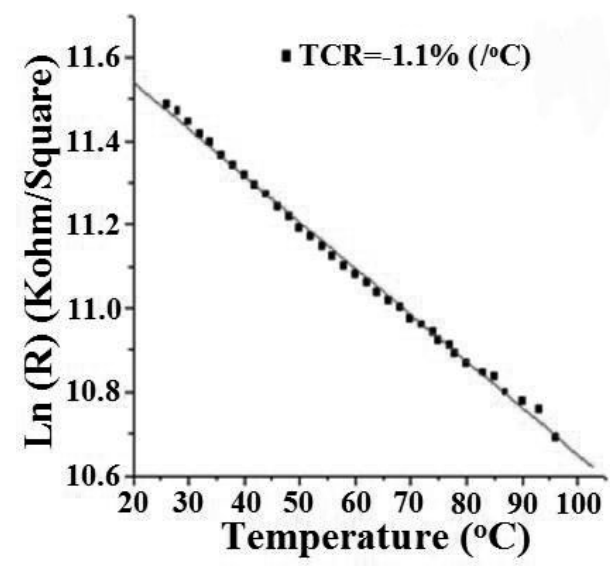

(a)

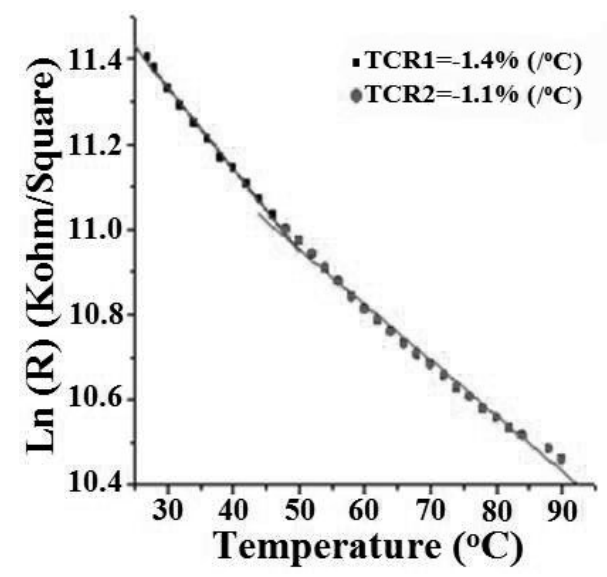

(b)

Fig. 4. Temperature-resistance relationship of nickel oxide thin films fabricated by ion beam sputtering and annealing at (a) $400^{\circ} \mathrm{C}$ for $2 \mathrm{~h}$ and (b) $500^{\circ} \mathrm{C}$ for $2 \mathrm{~h}$.

the TCR of the samples annealed at $400^{\circ} \mathrm{C}$, and the right figure (Fig. 4(b)) shows that of the samples annealed at $500^{\circ} \mathrm{C}$.

Unlike the as-deposited samples whose resistance increases versus temperature, the annealed samples show in-sheet resistance under heating. The TCR-temperature relationship changes into a negative value, which implies the formation of semiconducting materials. The left figure indicates a linear slope, but the right figure indicates a curve that is composed of two vectors. The TCR value in the right figure is 
$-1.1 \% /{ }^{\circ} \mathrm{C}$ and the TCR values in the left figure are -1.1 and $-1.4 \% /{ }^{\circ} \mathrm{C}$. In fact, the TCR values also reflect the relationship between temperature and the activation energy of the semiconductors; therefore, changes in TCR value at several temperatures indicate that the material is a mixture of several semiconducting phases, and the phases are related to the annealing temperature.

Figure 5 shows IR absorption spectra of the thin films annealed for $2 \mathrm{~h}$ in $\mathrm{O}_{2}$ ambient at different temperatures. The annealing temperatures were 400,500 , and $600^{\circ} \mathrm{C}$. From the spectra, it is observed that the thin films annealed at a higher temperature have a higher transmittance ratio within this spectral range (from 200 to $900 \mathrm{~nm}$ ). After 900 $\mathrm{nm}$, the transmittance curve is flat. The transmittance of the samples annealed at $600^{\circ} \mathrm{C}$ is above $80 \%$, which is shown as the uppermost curve in Fig. 5, and this transmittance is much higher than that of the samples annealed at a lower temperature. It is found that the material is sensitive to ultraviolet irradiation. Photons with wavelengths below $600 \mathrm{~nm}$ could be well absorbed using the thin films. The thin films clearly have semiconducting properties.

Another clear result that can also be observed in Fig. 5 is that the absorption edge of the samples annealed at $600^{\circ} \mathrm{C}$ is different from that of the samples annealed at 400 or $500^{\circ} \mathrm{C}$. The former is at about $300 \mathrm{~nm}$, while the latter is at about $450 \mathrm{~nm}$, although at about $300^{\circ} \mathrm{C}$, the edge is unconspicuous. This indicates that the annealing temperature affects the form of the material.

From the absorption curves, the energy band gap could also be calculated to be about $2.7 \mathrm{eV}$, which has been reported as the correct value of $\mathrm{NiO}_{x}$. In fact, the TCR values also reflect the values of the activation energy; therefore, the difference in TCR values means that the materials are in different semiconducting phases.

Figure 6 shows the measurements of gas sensitivity. The changes in sheet resistance in the samples exposed to air and $\mathrm{NH}_{3}$ flow at different working temperatures indicate the gas sensitivity of the nickel oxide thin films fabricated by ion beam sputtering and

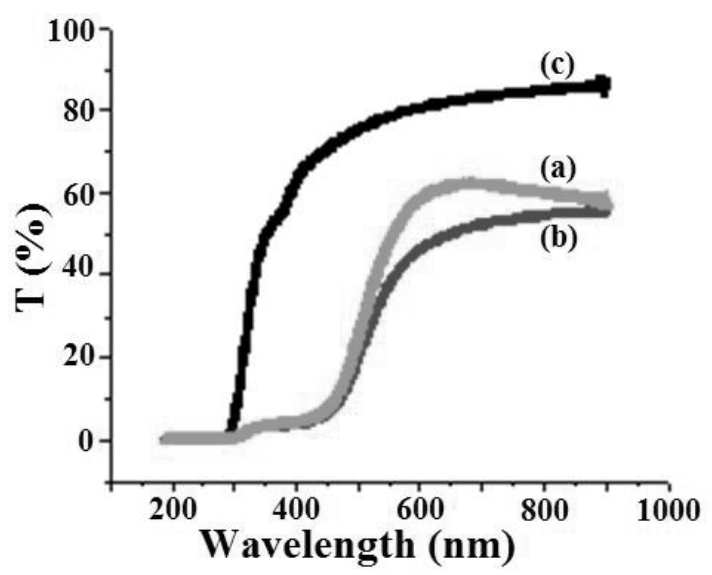

Fig. 5. IR absorption spectra of the thin films annealed for $2 \mathrm{~h}$ in $\mathrm{O}_{2}$ ambient at different temperatures: (a) 400 , (b) 500 , and (c) $600^{\circ} \mathrm{C}$. 


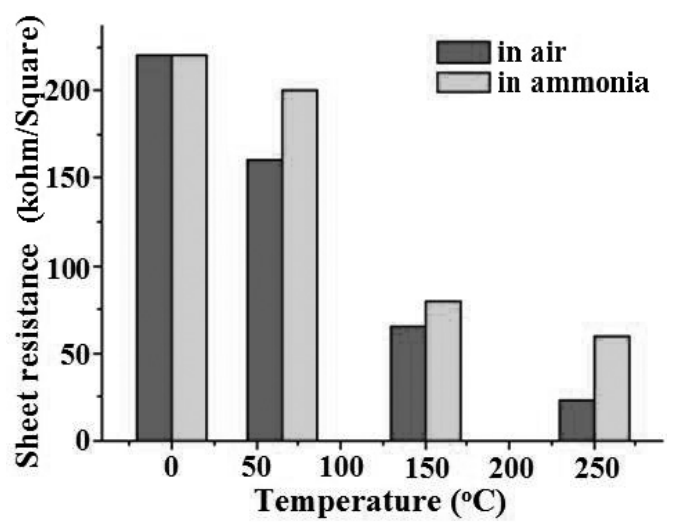

Fig. 6. Changes in sheet resistance of samples in $\mathrm{NH}_{3}$ flow at different working temperatures. The $\mathrm{NH}_{3}$ flux was $30 \mathrm{SCCM}$ in all cases.

annealing for $2 \mathrm{~h}$. It is found that the sheet resistance increases under the $\mathrm{NH}_{3}$ flow. While the $\mathrm{NH}_{3}$ gas flux is $30 \mathrm{SCCM}$ and the working temperature is about $65^{\circ} \mathrm{C}$, the sheet resistance changes from $163 \mathrm{k} \Omega /$ square (in air) to about $200 \mathrm{k} \Omega$ /square (in $\mathrm{NH}_{3}$ flow); when the $\mathrm{NH}_{3}$ flow is kept constant, the temperature increases to $150^{\circ} \mathrm{C}$, the sheet resistance changes from $72.5 \mathrm{k} \Omega /$ square (in air) to about $89 \mathrm{k} \Omega /$ square (in $\mathrm{NH}_{3}$ flow); when the temperature is changed to $250^{\circ} \mathrm{C}$, the sheet resistance changes from 25 $\mathrm{k} \Omega /$ square (in air) to about $62 \mathrm{k} \Omega / \mathrm{square}$ (in $\mathrm{NH}_{3}$ flow). As the difference between the sheet resistances in air and in $\mathrm{NH}_{3}$ flow increases, the sensitivity of the films increases. Therefore, it could be observed that the sensitivity increases with working temperature.

The histogram also reveals that the annealed films show a decrease in sheet resistance under heating in air or in ammonia, which indicates that the annealed films are formed by the semiconducting materials.

\subsection{Discussion}

The reason that we use an off-line oxidation process is that our deposition chamber cannot be treated at a temperature higher than $250^{\circ} \mathrm{C}$. The as-deposited thin films were obtained at low oxygen pressures at a temperature lower than $250^{\circ} \mathrm{C}$. This led to a lower oxygen content in the films.

Because the as-deposited nickel oxide films contain a lower oxygen concentration, they are typically of a metallic nature for which the resistance increases with temperature. After annealing, the oxidation degree of $\mathrm{NiO}_{x}$ was increased and the materials exhibited semiconducting characteristics where the resistance decreased with an increase in working temperature.

Typically, the oxygen concentration of $\mathrm{NiO}_{x}$ films markedly depends on the oxidation annealing conditions. During oxidation, oxygen diffuses into the as-deposited films and reacts with metallic nickel or its low valence oxides to form nickel oxide. The electrical homogeneity decreases throughout annealing, because the oxygen diffusion is nonisotropic. 
It is known that the as-deposited films are uniform and compact. For the annealed samples, the resistance of the samples was increased and oxygen-rich phases such as $\mathrm{NiO}$ were obtained. The resistance-temperature relationship changes into a negative value, which implies the formation of semiconducting materials. However, their homogeneity slightly decreases compared with that of the as-deposited samples. When the annealing temperature increases, the TCR value is not unique, which implies that the material of the thin film is a mixture of several semiconducting phases.

The changes in sheet resistance in the annealed nickel oxide thin films by ion beam sputtering in air and in $\mathrm{NH}_{3}$ flow at different working temperatures indicate that the films can be used as a gas sensor. The sensitivity of the nickel oxide films increases with the working temperature. As a result, choosing an appropriate working parameter is important.

\section{Conclusions}

In this paper, we have described a new method of fabricating $\mathrm{NiO}_{x}$ thin films that can be used as gas sensors. We fabricated $\mathrm{NiO}_{x}$ thin films by ion beam sputtering and oxidation annealing. By SEM observation, IR absorption, and TCR measurements, the fabricated materials were shown to be semiconductors with a perfect film surface. In addition, electrical measurements also indicated that different semiconducting phases are formed during the oxidation annealing process because of the difference in TCR value within different temperature ranges. The sensitivity measurements also indicated that the materials are sensitive to ammonia at a suitable working temperature.

\section{Acknowledgement}

This study was supported by the National Natural Science Foundation of China (No. 60607006), Scientific Research Foundation for Returned Scholars, Ministry of Education of China (No. 20081667), and Natural Science Foundation of Hubei Province of China (No. 2007ABA058).

\section{References}

1 M. Chigane and M. Ishikawa: Electrochim. 42 (1997) 1515.

2 K. S. Nanjundaswamy and M. S. Shankarshana Murthy: Mater. Chem. Phys. 15 (1986) 37.

3 M. Komath: Mater. Chem. Phys. 45 (1996) 171.

4 Y. M. Lu and W. S. Hwang: Surf. Coat. Technol. 155 (2002) 231.

5 P. S. Patil and L. D. Kadam: Appl. Surf. Sci. 199 (2002) 211.

6 Y. M. Lu, W. S. Hwang and Z. Q. Liu: Thin Solid Films 420 (2002) 54.

7 H. X. Hang, Q. F. Dong, X. H. Hu et al.: J. Mater. Sci. 79 (1999) 46.

8 F. C. Case: J. Vac. Sci. Technol. A 2 (1984) 1509.

9 K. S. Han and S. Tsurimoto: Solid State Ionics 121 (1999) 229.

10 S. B. Wang, S. B. Zhou and X. J. Yi: Vacuum 75 (2004) 85. 